\title{
Bases moleculares de la cancerización de cavidad oral
}

\author{
González-Moles MA*, Gil-Montoya JA*, Ruiz-Ávila I**
}

\section{RESUMEN}

Se presenta una revisión bibliográfica breve sobre los principales aspectos moleculares de interés en la cancerización de cavidad oral. Se hace referencia a los conocimientos más recientes sobre las aberraciones cromosómicas más comunes y las alteraciones de los oncogenes y genes supresores tumorales que están implicados en la carcinogénesis oral. Así mismo, se resume la teoría molecular actual que explica el proceso de cancerización de campo.

Palabras clave: Cáncer oral, Cavidad oral, Aspectos moleculares.

\section{SUMMARY}

A review about the main molecular aspects on oral cavity cancerization is presented, with special reference to the common chromosomal aberration, oncogenes and tumour suppressor genes implied in oral carcinogenesis. A summary about molecular theory explaining the field cancerization process is also presented.

Key words: Oral cancer, Oral cavity, Molecular aspects.

Fecha de recepción: Diciembre 2007.

Aceptado para publicación: Diciembre 2007.

* Departamento de Estomatología, Facultad de Odontología, Universidad de Granada. Campus de Cartuja sn, 18071. Granada. España.

** Servicio de Anatomía Patológica. Hospital General Universitario. Jaén. España.

González-Moles MA, Gil-Montoya JA, Ruiz-Ávila I. Bases moleculares de la cancerización de cavidad oral. Av. Odontoestomatol 2008; 24 (1): 55-60.

\section{INTRODUCCIÓN}

Actualmente se acepta que el cáncer oral es la consecuencia de la adquisición sumatoria de una serie de trastornos genómicos celulares que promueven la malignización a través de la activación directa o de la inactivación de genes que resultan claves para el mantenimiento del equilibrio celular normal. Este modelo multipaso de la carcinogénesis oral es ampliamente aceptado (1) y requiere la suma de eventos oncogénicos que dotan a las células epiteliales de ventajas proliferativas e invasivas.

Las alteraciones genéticas que acontecen en el cáncer oral se concretan, prioritariamente, en aberraciones cromosómicas, activación de oncogenes e inactivación de genes supresores tumorales. En este trabajo se discuten las más importantes y mejor conocidas y los conocimientos recientes en torno a cómo estas alteraciones pueden generar una predisposición al desarrollo de campos pre- 
malignos y de carcinomas múltiples de cavidad oral.

\section{ALTERACIONES GENÓMICAS}

\section{Aberraciones cromosómicas}

Son muchas las aberraciones cromosómicas que se pueden encontrar en el cáncer oral. Pueden aparecer ganancias o amplificaciones, o pérdidas de heterocigosidad $(\mathrm{LOH})$ o deleciones en determinados cromosomas. Algunas aberraciones aparecen precozmente y conllevan un valor pronóstico para los pacientes con lesiones premalignas o con carcinomas en estadios precoces. Determinados estudios comunican una alta prevalencia de LOH o deleciones en los loci cromosómicos 3p, 9p, 13q y 17p en lesiones orales premalignas y carcinomas precoces $(2,3)$. Estas regiones cromosómicas albergan importantes genes supresores tumorales cuya función preventiva del desarrollo tumoral probablemente se altera profundamente por la aberración cromosómica. El cromosoma 9 parece ser una de las dianas más precoces y sensibles en el desarrollo del cáncer habiéndose documentado pérdidas alélicas en la región 9p21 en la mayoría de las lesiones orales premalignas (4) y de los carcinomas incipientes $(3,5)$. La región 9p21 alberga genes que codifican los inhibidores de las kinasas dependientes de ciclinas p16 y 14, que actúan como importantes reguladores de la proliferación celular. Así mismo, algunas regiones del cromosoma 3, especialmente 3p25, 3p21 y 3p1314 , son frecuentes portadoras de aberraciones cromosómicas en el cáncer oral, aunque los genes que se alteran por estos imbalances alélicos son aun desconocidos (6). Otras aberraciones se asocian comúnmente a estadios tumorales avanzados o aparecen por lo general en carcinomas pobremente diferenciados. Ejemplos de ellos son las pérdidas alélicas en 5q21-22 (7), 22q13 (8), 4q, 11q, 18 q y 21 q (3).

\section{Oncogenes}

Los oncogenes son genes cuya activación genera un mecanismo molecular que colabora en la transformación maligna celular, prioritariamente a través de la promoción de la proliferación celular aberrante, de la inactivación de la maquinaria apoptótica celular o del establecimiento de mecanismos de supervivencia celular en condiciones adversas (9). Entre estos oncogenes, debido a su capacidad inherente para estimular el crecimiento de las células epiteliales, han recibido una especial atención aquellos que codifican los receptores de factores de crecimiento, singularmente la familia de receptores ErbB y en especial, el receptor del factor de crecimiento epidérmico (EGFR, también denominado ErbB1 o Her-1) (10). Las investigaciones sobre la utilidad clínica práctica del análisis de EGFR no han resultado concluyentes, aunque el empleo del anticuerpo monoclonal inhibidor de EGFR (gefitinib) en líneas celulares de cáncer oral y en pacientes con cáncer oral y de cabeza y cuello ha arrojado resultados esperanzadores en algunos casos (1113). Otros miembros de la familia ErbB también actúan como oncogenes. Por ejemplo, la amplificación de ErbB2 (también conocido como Her-2 o Neu) se ha podido observar en leucoplasias orales y cáncer (14-16), y algunos estudios han relacionado su sobreexpresión con un pobre pronóstico $(14,17)$. También, para este receptor se ha desarrollado un anticuerpo monoclonal (trastuzumab) que podría en el futuro incorporarse al arsenal terapéutico del cáncer oral en un subgrupo seleccionado de pacientes (9).

Los receptores de membrana implicados en el crecimiento celular y en otros procesos que podrían colaborar en la oncogénesis, ejercen sus acciones a través de señales de transducción dirigidas al núcleo celular mediante una variedad de mediadores que también se pueden alterar en algunos tipos de cánceres. Un ejemplo está constituido por los genes de la familia ras, integrada por los oncogenes H-, K- y N-ras. En modelos animales experimentales, la activación exclusiva de K-ras es suficiente para inducir la aparición de un cáncer oral (18). Sin embargo, la participación definitiva de estos oncogenes en el desarrollo del cáncer oral en humanos está por determinar y parece depender del área geográfica estudiada, con pocas influencias en países desarrollados (menos del $10 \%$ de mutaciones ras en USA, Europa y Japón) en comparación con las mutaciones encontradas en la India (35 \%) (19-22).

Los genes que codifican la síntesis de las proteínas ciclinas también pueden comportarse como oncogenes. Los miembros de esta familia activan el ciclo ce- 
lular. Las ciclinas tipo D, en respuesta a estímulos mitogénicos, promueven la fosforilación de la proteína del retinoblastoma (pRb), que inicia la transición de la fase $G$ a la fase $S$ del ciclo celular (23). El oncogén que la codifica, denominado CCND1, se encuentra frecuentemente amplificado en cáncer oral e induce una sobreexpresión de la proteína ciclina $\mathrm{D}$, que se relaciona con pobre pronóstico en tumores poco avanzados. Esta sobre expresión también se ha observado en lesiones premalignas $(24,25)$. Las ciclinas A y B igualmente se sobreexpresan en algunos carcinomas orales (26).

Un hecho determinante en la progresión tumoral es la angiogénesis. El fenómeno de producción de nuevos vasos sanguíneos a partir de los preexistentes dota al tumor de la capacidad de nutrirse y proliferar. Entre los mecanismos que regulan este proceso destacan los mediados por el factor de crecimiento vascular endotelial (VEGF). El gen que lo codifica puede comportarse como un oncogén en el cáncer oral. Los estímulos hipóxicos en el tejido tumoral disparan la producción de VEGF que actúa aumentando la microdensidad vascular $(27,28)$.

La movilidad de las células tumorales debe vencer la resistencia que ofrece el tejido sano circundante al tumor. Los mecanismos esenciales desplegados por las células neoplásicas en este sentido están mediados fundamentalmente por las acciones de las metaloproteinasas de matriz (MMP), un grupo de enzimas ricas en zinc que tienen la capacidad de degradar la matriz extracelular. Muchas de ellas se expresan en el cáncer oral y pueden jugar un papel en su progresión (29). La expresión inmunohistoquímica de MMP-2 y 9 se ha relacionado con el potencial invasivo, y MMP2 parece estar asociada también a capacidad metastatizante a ganglios linfáticos (29). La expresión de estas metaloproteinasas se ha correlacionado igualmente con el consumo de alcohol, lo que ha inducido a determinados autores a teorizar sobre la posibilidad de que la participación del alcohol en la carcinogénesis oral se realice a través de estímulo de estas MMPs (9).

\section{Genes supresores tumorales}

Son genes cuya activación protege a la célula de la adquisición de características malignas, actuando a nivel de diferentes puntos de chequeo del ciclo celu- lar. La proteína del retinoblastoma (pRb) y su sistema molecular asociado se encuentran frecuente y precozmente alterados en el cáncer oral. El estado hipofosforilado de $\mathrm{pRb}$ y de otros miembros de su familia, como p107 y p130, permite que activen al factor de trascripción E2F que resulta determinante para la progresión del ciclo celular desde la fase $\mathrm{G}$ a la fase $\mathrm{S}$. En un alto porcentaje de lesiones orales premalignas (64\%) y de carcinomas orales $(70 \%)$ se puede demostrar por inmunohistoquímica una ausencia de expresión de pRb, lo que se interpreta como una falta paralela de las funciones reguladoras del ciclo ligadas a esta proteína $(30,31)$. Además, la mayoría de los carcinomas orales presentan una expresión alterada de al menos uno de los miembros del sistema pRb (32).

Los genes que codifican las proteínas inhibidoras de las quinasas dependientes de ciclinas (CDKIs) se comportan como importantes genes supresores tumorales, promoviendo un adecuado funcionamiento de $\mathrm{pRb}$ y por tanto un correcto control del ciclo celular. El gen CDKN2A, que codifica la proteína p16, se localiza en el locus 9p21, una de las áreas más vulnerables del genoma humano en el cáncer oral. En un altísimo porcentaje de carcinomas orales (más del $80 \%$ ) se demuestra una falta de función de p16 $(30,33,34)$, lo que resalta la importancia de esta proteína en el mantenimiento de una homeostasis celular normal. De igual forma, el trascrito alternativo del gen CDKN2A, p14, está también frecuentemente suprimido en lesiones orales malignas (34).

Uno de los genes supresores tumorales más importantes en el ser humano es el TP53. La supresión de las funciones de este gen y su sistema molecular asociado se observa en numerosos tumores humanos y constituye uno de los hallazgos más precoces en la historia natural del cáncer $(35,36)$. La proteína p53 es capaz de detener el ciclo celular en respuesta a lesiones del ADN para establecer los mecanismos necesarios para su reparación, y en caso de lesiones irreparables del ADN, p53 induce apoptosis. Estos procesos mediados por el sistema p53 eliminan la posibilidad de que se perpetúen estirpes celulares con ADN lesionado en las que nuevos eventos oncogénicos sumatorios puedan concluir con el desarrollo de un fenotipo maligno. Entre el 50-70\% de los carcinomas orales expresan proteína p53 por métodos inmunohistoquímicos, lo que se interpreta como una alteración en la 
estructura con pérdida de función de la proteína, un bloqueo de la proteína por parte de oncoproteínas víricas o un mecanismo de escape de otra naturaleza a las acciones antitumorales ejercidas por p53 $(37,38)$.

\section{Teoría de la cancerización de campo}

El concepto de cancerización de campo fue inicialmente propuesto por Slaughter en 1953 (39) en un intento de explicar la frecuente aparición de nuevos tumores en vía aerodigestivas superiores de pacientes que padecen un primer tumor de cavidad oral u orofaringe. El conocimiento actual de los mecanismos moleculares implicados en la regulación del ciclo celular ha posibilitado dar una interpretación molecular a este fenómeno y definir de forma más precisa la naturaleza de los segundos tumores que aparecen en algunos pacientes. Los eventos oncogénicos moleculares más precoces en el desarrollo del cáncer oral son las mutaciones del gen TP53 y la pérdida de heterocigosidad ( $\mathrm{LOH}$ ) en los loci cromosómicos 3p, 9p y 17p. Estas regiones cromosómicas albergan importantes genes reguladores del ciclo celular. La consecuencia de las alteraciones genéticas precoces será que la célula alterada adquiere una ventaja proliferativa sobre el resto de las células del epitelio de la mucosa oral. Se crea así un campo precanceroso expansivo que va progresivamente reemplazando al epitelio oral normal. Todas las células de este campo precanceroso comparten las mismas alteraciones genéticas precoces. Por definición, los campos precancerosos son expansivos aunque no invasivos. No han adquirido aun la capacidad de atravesar la membrana basal del epitelio y acceder al corion, lugar en el que el proceso metastáticos es posible. Esta capacidad invasiva define al cáncer y marca la diferencia entre campo precanceroso y cáncer. La capacidad invasiva se adquiere presumiblemente por la suma de eventos oncogénicos más tardíos, como son las alteraciones en los loci cromosómicos 8p, 13q y 18q. Posiblemente, también otras alteraciones genómicas ligadas a la perdida de expresión y función de las moléculas de adhesión, se asocian a la adquisición de capacidad invasiva por parte de las células del campo precanceroso (9).

La definición de las alteraciones oncogénicas que aparecen el los campos precancerosos ha permitido conocer más profundamente la naturaleza de los tumores múltiples que aparecen en cavidad oral y orofaringe en algunos pacientes. Segundos tumores primarios serían aquellos que tienen un perfil genético completamente diferente al tumor inicial. Los segundos tumores de campo serían los que comparten con el tumor inicial las alteraciones genéticas precoces del campo precanceroso (mutaciones de TP53 y LOH 3p, 9p y 17p), pero no las alteraciones tardías (LOH en 8p,13q y 18q). En este tipo de tumores, las alteraciones genéticas precoces del campo han posibilitado la existencia de un idéntico sustrato genéticamente alterado en todas las células del campo en la que distintos eventos tardíos generan carcinomas sobre el campo precanceroso, solo parcialmente diferentes. Una metástasis se define como la aparición de nuevo tejido tumoral con un patrón idéntico al tumor inicial y localizado a distancia del tumor primario. Finalmente, una recurrencia es la aparición de nuevo tejido tumoral con un patrón genético idéntico al del tumor inicial y localizado en la misma región anatómica. (32,40-42).

\section{BIBLIOGRAFÍA}

1. Fearon ER, Vogelstein B. A genetic model for colorectal tumorigenesis, Cell 1990; 61:759-67.

2. Rosin MP, Cheng X, Poh C, Lam WL, Huang Y, Lovas $\mathrm{J}$, et al. Use of allelic loss to predict malignant risk for low-grade oral epithelial dysplasia. Clin Cancer Res 2000;6:357-62

3. Bockmühl G, Wolf S, Schmidt A, Schwendel V, Jahnke V, Dietel M, et al. Genomic alterations associated with malignancy in head and neck cancer. Head Neck 1998;20:145-51

4. Jiang WW, Fujii H, Shirai H, Mega H, Takagi M. Accumulative increase of loss of heterozygosity from leukoplakia to foci of early cancerization in leukoplakia of the oral cavity. Cancer 2001;92:2349-56.

5. el Naggar AK, Hurr K, Batsakis JG, Luna MA, Goepfert H, Huff V. Sequential loss of heterozygosity at microsatellite motifs in preinvasive and invasive head and neck squamous carcinoma. Cancer Res 1995; 55: 2656-9.

6. Partridge M, Emilion G, Pateromichelakis S, A'Hern R, Lee G, Phillips E, et al. The prognostic 
significance of allelic imbalance at key chromosomal loci in oral cancer. $\mathrm{Br} \mathrm{J}$ Cancer 1999;79:1821-7

7. Mao EJ, Schwartz SM, Daling JR, Beckmann AM. Loss of heterozygosity at 5q21-22 (adenomatous polyposis coli gene region) in oral squamous cell carcinoma is common and correlated with advanced disease. J Oral Pathol Med 1998; 27:297-02.

8. Reis PP, Rogatto SR, Kowalski LP, Nishimoto IN, Montovani JC, Corpus G, Squire JA, Kamel-Reid S. Quantitative real-time PCR identifies a critical region of deletion on 22q13 related to prognosis in oral cancer. Oncogene 2002;19:6480-7.

9. Tsantoulis PK, Kastrinakis NG, Tourvas AD, Laskaris G, Gorgoulis VG. Advances in the biology of oral cancer.Oral Oncol 2007;43:523-34.

10. Normanno N, De Luca A, Bianco C, Strizzi L, Mancino M, Maiello MR, Carotenuto A, De Feo G, Caponigro F, Salomon DS. Epidermal growth factor receptor (EGFR) signaling in cancer. Gene 2006;17:2-16.

11. Shintani S, Li C, Mihara M, Nakashiro K, Hamakawa H. Gefitinib ('Iressa'), an epidermal growth factor receptor tyrosine kinase inhibitor, mediates the inhibition of lymph node metastasis in oral cancer cells. Cancer Lett 2003;25:149-55.

12. Cohen EE, Rosen F, Stadler WM, Recant W, Stenson K, Huo D, Vokes EE. Phase II trial of ZD1839 in recurrent or metastatic squamous cell carcinoma of the head and neck. J Clin Oncol 2003;15:1980-7.

13. Kirby AM, A'Hern RP, D'Ambrosio C, Tanay M, Syrigos KN, Rogers SJ, Box C, Eccles SA, Nutting CM, Harrington KJ. Gefitinib (ZD1839, Iressa) as palliative treatment in recurrent or metastatic head and neck cancer. Br J Cancer 2006;13: 631-6.

14. Xia W, Lau YK, Zhang HZ, Liu AR, Li L, Kiyokawa N, Clayman GL, Katz RL, Hung MC. Strong correlation between c-erbB-2 overexpression and overall survival of patients with oral squamous cell carcinoma. Clin Cancer Res 1997;3:3-9.

15. Werkmeister R, Brandt B, Joos U. Aberrations of erbB-1 and erbB-2 oncogenes in non-dysplastic leukoplakias of the oral cavity. Br J Oral Maxillofac Surg 1999;37:477-80.

16. Chen YJ, Lin SC, Kao T, Chang CS, Hong PS, Shieh TM, Chang KW. Genome-wide profiling of oral squamous cell carcinoma. J Pathol 2004;204: 326-32.

17. Werkmeister R, Brandt B, Joos U. Clinical relevance of erbB-1 and -2 oncogenes in oral carcinomas. Oral Oncol 2000;36:100-5.

18. Caulin C, Nguyen T, Longley MA, Zhou Z, Wang $\mathrm{XJ}$, Roop DR. Inducible activation of oncogenic K-ras results in tumor formation in the oral cavit 0079. Cancer Res 2004;64:5054-8.

19. Xu J, Gimenez-Conti IB, Cunningham JE, Collet AM, Luna MA, Lanfranchi HE, Spitz MR, Conti CJ. Alterations of p53, cyclin D1, Rb, and H-ras in human oral carcinomas related to tobacco use. Cancer 1998;83:204-12.

20. Kiaris H, Spandidos DA, Jones AS, Vaughan ED, Field JK. Mutations, expression and genomic instability of the H-ras proto-oncogene in squamous cell carcinomas of the head and neck. Br J Cancer 1995;72:123-8.

21. Sakata K. Alterations of tumor suppressor genes and the H-ras oncogene in oral squamous cell carcinoma. J Oral Pathol Med. 1996 Jul;25 (6):302-7.

22. Matsuda H, Konishi N, Hiasa Y, Hayashi I, Tsuzuki T, Tao M, Kitahori Y,Yoshioka N, Kirita T, Sugimura M. Alterations of p16/CDKN2, p53 and ras genes in oral squamous cell carcinomas and premalignant lesions. J Oral Pathol Med 1996; 25:232-8.

23. Sherr CJ, Roberts JM. CDK inhibitors: positive and negative regulators of G1-phase progression. Genes Dev 1999;13:1501-12.

24. Rousseau A, Lim MS, Lin Z, Jordan RC. Frequent cyclin D1 gene amplification and protein overexpression in oral epithelial dysplasias. Oral Oncol 2001;37:268-75.

25. Myo K, Uzawa N, Miyamoto R, Sonoda I, Yuki Y, Amagasa T. Cyclin D1 gene numerical aberration is a predictive marker for occult cervical lymph node 
metastasis in TNM Stage I and II squamous cell carcinoma of the oral cavity. Cancer 2005;104:2709-16.

26. Kushner J, Bradley G, Young B, Jordan RC. Aberrant expression of cyclin A and cyclin B1 proteins in oral carcinoma. J Oral Pathol Med 1999; 28:77-81.

27. Shang ZJ, Li ZB, Li JR. VEGF is up-regulated by hypoxic stimulation and related to tumour angiogenesis and severity of disease in oral squamous cell carcinoma: in vitro and in vivo studies, Int $\mathrm{J}$ Oral Maxillofac Surg 2006;35:533-8.

28. Li C, Shintani S, Terakado N, Klosek SK, Ishikawa T, Nakashiro K et al. Microvessel density and expression of vascular endothelial growth factor, basic fibroblast growth factor, and platelet-derived endothelial growth factor in oral squamous cell carcinomas, Int J Oral Maxillofac Surg 2005;34: 559-65.

29. Thomas GT, Lewis MP, Speight PM. Matrix metalloproteinases and oral cancer, Oral Oncol 1999;35:227-33.

30. Pande P, Mathur M, Shukla NK, Ralhan R. pRb and p16 protein alterations in human oral tumorigenesis. Oral Oncol 1998;34:396-403.

31. Sartor M, Steingrimsdottir H, Elamin F, Gäken J, Warnakulasuriya S, Partridge $M$, et al. Role of p16/ MTS1, cyclin D1 and RB in primary oral cancer and oral cancer cell lines, Br J Cancer 1999;80: 79-86.

32. Soni S, Kaur J, Kumar A, Chakravarti N, Mathur $\mathrm{M}$, Bahadur $\mathrm{S}$, et al. Alterations of Rb pathway components are frequent events in patients with oral epithelial dysplasia and predict clinical outcome in patients with squamous cell carcinoma. Oncology 2005;68:314-25.

33. Reed AL, Califano J, Cairns P, Westra WH, Jones RM, Koch W, et al. High frequency of p16 (CDKN2/MTS-1/INK4A) inactivation in head and neck squamous cell carcinoma, Cancer Res 1996;56:3630-3.

34. Wu CL, Roz L, Mckown S, Sloan P, Read AP, Holland S, et al. DNA studies underestimate the major role of CDKN2A inactivation in oral and oropharyngeal squamous cell carcinomas. Genes Chromosomes Cancer 1999;25:16-25.
35. Gorgoulis VG, Vassiliou LVF, Karakaidos P, Zacharatos P, Kotsinas A, Liloglou T, et al. Activation of the DNA damage checkpoint and genomic instability in human precancerous lesions. Nature 2005;434:907-13.

36. Bartkova J, Horejs Z, Koed K, Krmer A, Tort F, Zieger $\mathrm{K}$, et al. DNA damage response as a candidate anti-cancer barrier in early human tumorigenesis. Nature 2005;434:864-70.

37. Ogden GR, Kiddie RA, Lunny DP, Lane DP. Assessment of $\mathrm{p} 53$ protein expression in normal, benign, and malignant oral mucosa. J Pathol 1992:389-94.

38. De Vicente JC, Gutiérrez LMJ, Zapatero AH, Forcelledo MFF, Hernández-Vallejo G, Arranz JSL. Prognostic significance of p53 expression in oral squamous cell carcinoma without neck node metastases. Head Neck 2004;283-93.

39. Slaughter DP, Southwick HW, Smejkal W. Field cancerization in oral stratified squamous epithelium; clinical implications of multicentric origin. Cancer 1953;6:963-8.

40. Braakhuis BJM, Tabor MP, Kummer JA, Leemans CR, Brakenhoff RH. A genetic explanation of slaughter's concept of field cancerization: evidence and clinical implications. Cancer Res 2003;63:1727-30.

41. Braakhuis BJM, Leemans CR, Brakenhoff RH. A genetic progression model of oral cancer: current evidence and clinical implications. J Oral Pathol Med 2004;33:317-22

42. Tabor MP, Brakenhoff RH, Ruijter-Schippers HJ, Wal JE, SnowGB, Leemans CR, et al. Multiple head and neck tumors frequently originate from a single preneoplastic lesion. Am J Pathol 2002;161:1051-60.

\section{CORRESPONDENCIA}

Miguel Ángel González Moles.

Departamento de Estomatología.

Facultad de Odontología. Universidad de Granada.

Campus de Cartuja, s/n,

18071. Granada. España.

E-mail:magonzal@ugr.es 